UAM Vol. 31 Pozrañ 2004

\author{
JOANNA WLLK-RACIESKA
}

Universidad de Silesia

\title{
"VERDES IDEAS SIN COLOR NO PUEDEN DORMIR FURIOSAMENTE" \\ (RESTRICCIONES QUE LOS CONCEPTOS [PREDICADOS] IMPONEN SOBRE SUS ARGUMENTOS)
}

\begin{abstract}
Wilk-Racięska Joanna, "Verdes ideas sin color no pueden dormir furiosamente" (Restricciones que los conceptos (predicados] imponen sobre sus argumentos) [Green ideas with no colour cannot sleep furiously. The restrictions that concepts (predicates) impose upon their arguments]. Studia Romanica Posnaniensia, Adam Mickiewicz University Press, Poznań, vol. XXXI: 2004, pp. 469475. ISBN 83-232-1353-4, ISSN 0137-2475.
\end{abstract}

The purpose of this paper is a short presentation of some types of restrictions imposed by predicates upon the objects selected as their own arguments. We investigate several grammar procedures used by languages in order to classify objects in argument positions. We will also discuss the relations between these selections and different cultural schemes which can affect the classification and determine the use of linguistic forms. Theoretical instrument applied is the semantic theory known as semantic grammar or semantic syntax.

Nuestro objetivo es presentar brevemente las dependencias que existen entre las estructuras predicativas, las propiedades selectivas de las posiciones de argumentos y los diferentes modos de explicitar aquellas relaciones en el nivel formal-comunicativo (estudiadas, por lo general, en el marco de «valencia») de una parte, y de la otra, las bases socio-culturales que crean la visión del mundo y, en consecuencia, influyen en el lenguaje de una comunidad socio-lingüística dada.

Sin embargo, no intentaremos todavía analizar profundamente ni clasificar el fenómeno. Nos limitaremos simplemente a presentar un puñado de observaciones, enfocando de un modo especial la cuestión de las restricciones que los conceptos [predicados] imponen a los objetos seleccionados como sus argumentos. Empecemos por una verdad elemental, casi trivial: la lengua sirve para comunicarnos entre nosotros y por tanto nos ofrece diferentes estrategias para destacar lo que queremos destacar y ocultar o simplemente omitir lo que no nos gusta comunicar. 
La manera de describir el mundo depende solamente de nosotros mismos, de nuestro modo de ver y experimentarlo. La lengua puede entregarnos numerosas herramientas para hacerlo porque cada visión del mundo se las ha creado. Incluso en el marco de la misma comunidad socio-lingüística los flemáticos experimentan el mundo de un modo diferente que los coléricos. Comparemos dos situaciones:

1. Se escaparon todas las tortugas de un zoológico. El director pregunta a la persona responsable por estos animales: “¿Pero cómo ha podido pasar eso?", "Señooor - responde el guarda - nooo logrééé paraaarlas... Se abrieeeron las jaaaulas y las tortuuugas se fueeeron en seguiiida..."

2. En una carretera tuvo lugar una colisión frontal de dos apisonadoras (Como es bien sabido, aquellas máquinas se mueven muy lentamente). Vienen los guardias y uno pregunta muy sorprendido: “¿Pero, hombre, cómo ha podido pasar eso?” Uno de los conductores baja rápidamente de la máquina, se acerca al guardia y dice: "Pues, fíjate hombre, ;fue un momento!"

Pasaremos ahora a la cuestión de las propiedades selectivas de los predicados o, simplemente, las restricciones que el predicado impone a sus posiciones para los argumentos.

Estamos aquí ante el problema de la aceptabilidad/inaceptabilidad semántica frente a la aceptabilidad/inaceptabilidad gramatical.

Se admite, por lo general, que los enunciados de una lengua dada son fácilmente identificables como tales por sus hablantes nativos y por las personas competentes en esta lengua.

Podemos, pues, tranquilamente advertir que en español no se aceptará ninguno de los siguientes enunciados: "Verdes ideas sin color duermen furiosamente"; "Quiero que Pedro lo hace". Haciendo abstracción de la poesía u otros "fines específicos' que nos permiten interpretar el primero de los ejemplos anteriores en los contextos adecuados, observemos la diferencia entre los dos enunciados: el primero es universalmente inaceptable, a pesar de su buena formación gramatical, mientras que el otro representa "un mal recipiente para un buen contenido". En este enunciado no hay nada que le haga incorrecto en lo que parece ser el significado buscado. Para obtener su versión correcta, basta simplemente con aplicarle las reglas sintácticas del español que permitirán volverlo gramatical.

Sin embargo, para entender el primer enunciado ya no basta con aplicarle las reglas idiomáticas del español. Por este motivo concentraremos ahora la atención en algunas reglas semánticas que dirigen la estructura de la proposición y en consecuencia permiten la formación de las proposiciones semánticamente correctas.

En la lingüística contemporánea se utiliza en este caso la denominación de «valencia» la cual cubre por lo general tanto la cuestión del número y tipo de posiciones implicadas por los predicados como las propiedades semánticas de los argumentos que ocupan estas posiciones.

Entre estas últimas englobamos tales propiedades como: animado/inanimado; personal/impersonal o el número sing./pl. y algunas otras que los argumentos deben 
poseer para poder saturar las posiciones implicadas por los predicados. Presentemos unos ejemplos (S. Karolak, 1991).

Uno de los predicados que menos restricciones parece imponer a sus argumentos es el predicado de localización [ $\mathrm{f}(\mathrm{x}, \mathrm{y})$ ] representado por expresiones tales como hallarse, estar, situarse, etc. Cualquier objeto puede hallarse en cualquier sitio: "El perro está debajo de la cama" o "Su programa personal está ya dentro del ordenador". Tampoco son inaceptables, aunque a veces un poco raras por falta de experiencia, proposiciones como: "El gato está en la sopa", que es simplemente una cuestión de cantidad.

El predicado de posesión $[\mathrm{f}(\mathrm{x}, \mathrm{y})]$ impone una sola restricción a su primer argumento: que debe ser animado aunque no necesariamente personal. Por suerte, el segundo argumento del predicado de posesión ya no parece estar sometido a ninguna restricción: uno puede poseer cualquier cosa. Por ejemplo, he leído que una actriz ha comprado un planeta para el cumpleaños de su marido. Así, este señor tiene/posee un planeta.

Los predicados como ir o correr requieren en la primera posición de los argumentos animados o automáticos. Sin embargo, esta restricción no ataña todos los predicados de movimiento como, p.e. saltar donde las exigencias no son tan rígidas: saltan las personas y los peces en el río pero también las pelotas y las damas en el ajedrez.

En la primera posición de los predicados de opinión, solo se aceptan los argumentos personales: pensar, opinar.

En cuanto a los predicados de orden superior, observamos que algunos de ellos imponen restricciones en la posición del argumento proposicional. Por ejemplo, el predicado de decisión no acepta en esta posición proposiciones constituidas por conceptos que signifiquen propiedades independientes de la voluntad del hombre (amor, odio, celos):

"*Manolo ha decidido odiar a Paula" vs.: "Manolo ha decidido dejar de fumar"'.

Podríamos terminar en este sitio concluyendo que los factores responsables de la formación de una proposición semánticamente correcta son, en primer lugar, la estructura predicativa representada por una expresión dada, el grado de complejidad

${ }^{1}$ Hay también otros tipos de restricciones como las restricciones temporales [querer y saber, *Antonio quiere que Maria hubiera venido vs. Antonio quiere que Maria venga y Antonio sabe que María ha llegado; Antonio sabe que esto es verdad vs.: *Antonio sabe que María vendrá mañana]; las restricciones de correferencia. En este grupo nos encontramos con tres situaciones:

1) correferencia facultativa: querer, decidir: Manolo quiere que Ana salga vs. *Manolo quiere que salga (él mismo);

2) correferencia obligatoria: prometer: Manolo prometió que vendría vs.: Manolo prometió que Ana vendría que también es aceptable, pero exclusivamente como el resultado de una condensación: Manolo prometio hacer algo para que Ana viniera;

3) exclusión de correferencia: tolerar: *Manolo no tolera que esté enfermo (él mismo). 
de esta estructura y, en consecuencia las restricciones que el predicado impone a sus argumentos, mientras que el modo de explicitar los sentidos, de revelar o bloquear las posiciones de los argumentos, en total, de elegir las palabras y su orden adecuado son, ante todo, cuestión del nivel comunicativo.

No obstante, la cuestión no es tan simple como a primera vista parece. Algunas de las propiedades de los predicados parecen ser universales y no discutibles como p.e. la «estatividad» de los predicados que denotan la existencia, la permanencia frente a la «dinamicidad» de los que denotan acciones. De estas propiedades predicativas resultan directamente algunas restricciones impuestas a las posiciones de sus argumentos.

Tomemos como ejemplo ilustrativo el concepto de control y el de agente considerados comúnmente como inherentes a la estructura conceptual representada por un grupo muy amplio de verbos (ir, escribir, patear, pegar, etc.).

Según la teoría el predicado de acción [ $\mathrm{f}(\mathrm{x}, \mathrm{y})]$ requiere en la primera posición, ante todo de argumentos animados pero acepta también objetos automáticos, es decir, programados o conducidos por el hombre, y que el segundo argumento implicado por este concepto no parece estar sometido a ninguna restricción.

Vamos a verificar estas observaciones en unos "mundos" un tanto diferentes del nuestro.

Por ejemplo, parece obvio que si "Pedro está golpeando a Pablo", "Pedro" es el agente y, a la vez, (si, en este momento, no está ciego de cólera) evidentemente puede controlar la situación.

Por otra parte, el predicado patear rige un ejecutante de la acción en la primera posición, pero no es necesario que éste controle la situación.

En "El caballo está pateando/pateó a un hombre", el caballo no parece haber decidido conscientemente hacerlo. También es evidente que, para los fines puramente informativos no decimos "?Esta dama está devorando un chocolate" porque, aunque devorar reserva la posición del primer argumento para los objetos animados, estos objetos deben ser, más bien, salvajes y las damas por definición no pertenecen a esta categoría (J. Wilk-Racięska, en prensa). En consecuencia, la oración "Un león está devorando a una dama" sería ya semánticamente aceptable en nuestra cultura, aunque toda la acción estaría (para nosotros) fuera del control del animal.

No obstante, como han probado numerosos investigadores de las culturas indígenas lo que para las lenguas europeas (Creider, 1975; Lan, 1985; Spitulnik, 1987; Palmer, 2000;) parece ser obvio y universal no siempre es tal para otras sociedades socio-lingüísticas. Palmer (2000: 164) afirma que los términos protobantúes para «jefe», «hechicero» y «persona ciega» aparecen en la clase animal y no en la humana. "¿Es esto porque de alguna manera son más salvajes que las personas normales o porque están menos sometidos al control de los humanos corrientes?" - pregunta el lingüista. 
Lan (1985) explica esta clasificación aludiendo a la cultura de los shona de Zimbabwe. Los espíritus de los jefes ancestrales [mhondoro] viven aquí en los cuerpos de los leones y reinan sobre las cosas del mundo salvaje y sobre los asuntos humanos. Los leones mondhoro no atacan a las personas sino que atraen la lluvia pero la lengua no hace diferencia entre los leones auténticos y los espíritus; mondhoro significa león. Así las cosas, en esta cultura sería un poco raro que un león devorase a una dama pero si el mismo león devorase una vaca o un pollo (que suelen matarse y comerse durante los rituales ceremoniales), lo haría con plena conciencia y control de la situación.

En cambio, la oración "El caballo pateó a un hombre" totalmente aceptable en nuestra cultura (naturalmente, desde un punto de vista semántico) no es aceptable en navajo. Como advierte G. Palmer (1996: 184-192), en navajo, lengua de una cultura que se supone vivir en un mundo animado, existen dos tipos de agentes: aquellos que controlan a otros agentes y aquellos que carecen de control. En la visión del mundo de los navajos, nada sucede sin la intención y el control de algún ser animado, un ser humano o un espíritu. Hay una jerarquía de control, de modo que los seres animados pueden controlar a los inanimados, pero no a la inversa. No obstante, entre los seres animados también hay una jerarquía de control según la potencia e inteligencia relativas del agente y del paciente. Por este motivo, aunque los navajos pueden decir "El hombre pateó el caballo", la cláusula "*?El caballo pateó al hombre" es inaceptable (es "mal navajo"), porque viola el conocimiento cultural navajo de cómo se controlan unas a otras las entidades. La segunda cláusula puede, entonces, expresarse como: "El hombre se dejó patear por el caballo", donde el control viene marcado con un prefijo especializado. De ahí resulta que la idea de control, aunque presente en la semántica del navajo, no se deja someter facílmente a los criterios lingüísticos aplicados a lenguas como, p.e., el español.

En nuestras lenguas (el español o el polaco) el predicado «patean» implica un ser animado ejecutante de la acción en una de las posiciones del argumento pero no precisa si este agente sólo ejecuta la acción o bien, al mismo tiempo, la dirige y controla. En cambio, los predicados de acción en navajo parecen tener incorporada la ídea de control unidireccional, la cual sólo se deja suprimir a través del uso de un exponente de otro predicado de control (los prefijos especializados).

Dicho de otro modo, la oración "El caballo pateó al hombre" sería aceptable bajo la condición de que el caballo fuera más poderoso que el hombre. A modo de consolación podemos decir que "El caballo pateó al mulo" es perfectamente aceptable (Witherspoon, 1977; Palmer, 2000: 184) probablemente porque el mulo es menos inteligente...

El navajo parece también poner en evidencia una oposición más que para nosotros no es nada dudosa. Es la oposición entre «estatividad» y «dinamismo». Los conceptos de «estatividad» y de «dinamismo» están, a nuestro parecer, presentes en la semántica de los predicados adecuados y esta presencia impone algunas restricciones a las posiciones de los argumentos como, p.e., la incompatibilidad de 
los predicados de movimiento o transición con los objetos de algún modo permanentes: "*La columna se ha ido de la plaza"; "*Pedro está mortal", etc.

Como observan los investigadores [Hoijer (1964), Witherspoon (1977) o Palmer (2000), por citar sólo algunos] "el mundo navajo es un lugar donde todas las cosas ya se han puesto en movimiento" (Palmer, 2000: 184).

Según Hoijer (1964a: 146) "los verbos navajos expresan movimiento con un detalle minucioso, incluso hasta el punto de clasificar como semánticamente diferentes los movimientos de uno, dos o más cuerpos." Este modo de ver el mundo afecta también a aquellos verbos que a primera vista no parecen expresar el movimiento y a los sustantivos que se definen en términos de "alguna acción o movimien-to característico de un objeto o conjunto de objetos" (Hoijer, en Palmer, 2000: 183).

Basta con citar algunos ejemplos: la palabra que significa «luna llena» se traduce como «un objeto en forma de aro ha girado»; «papel», «carta» = «un objeto parecido a tela se mueve».

Todo ello parece implicar interesantes diferencias entre las propiedades selectivas de "nuestros" predicados y el tipo de restricciones que los predicados navajos imponen a las posiciones de sus argumentos, las cuales quedan por investigar

Ahora bien, si añadimos aquí las lenguas tales como el chino que no dispone del tiempo gramatical hecho que no parece, sin embargo, sugerir que esta lengua carezca de restricciones estrictamente temporales, y en cambio, dispone de medios para expresar objetos sin especificar su número (Comrie), nos hallaremos ante un campo de investigación muy interesante.

La complejidad de estructuras predicativas, las propiedades selectivas de los argumentos $y$, por fin, los modos de explicitarlos en una lengua dada están vinculados por relaciones muy complejas y, a veces, inesperadas, con la cultura que ha creado la lengua.

Visto lo dicho, la característica de Tlön y de su lenguaje hecha por Jorge Luis Borges en sus Ficciones no nos parece del todo imposible:

Las naciones de este planeta son - congénitamente - idealistas. Su lenguaje y las derivaciones de su lenguaje - la religión, las letras, la metafísica - presuponen el idealismo. El mundo para ellos no es un concurso de objetos en el espacio; es una serie heterogénea de actos independientes. Es sucesivo, temporal, no espacial. No hay sustantivos en la conjetural Ursprache de Tlön, de la que proceden los idiomas "actuales" y los dialectos: hay verbos impersonales, calificados por sufijos (o prefijos) monosilábicos de valor adverbial. Por ejemplo no hay palabra que corresponda a la palabra «luna», pero hay un verbo que sería en español «lunecer». Surgió la luna sobre el rio se dice hlör u fang axaxaxas milö o sea en su orden: hacia arriba detrás duradero - fluir luneció" (J. Borges, 1956, Ficciones, «Tlön, Uqbar, Orbis Tertius» EMECÉ, Buenos Aires).

\footnotetext{
${ }^{2}$ Véase tambićn Palmer, 2000: 180-184.
} 
La «lingüística cultural» se ha desarrollado mucho últimamente. Sin embargo, no podemos olvidar que ya Wittgenstein observó que "los límites de nuestro lenguaje significan los límites de nuestro mundo".

\section{REFERENCIAS BIBLIOGRÁFICAS}

Bogacki, K., Karolak, S. (1991), Fondements d'une grammaire à base sémantique, Lingua e Stile, a. XXVI, n. 3, settembre 1991, 309-345.

Creider, Ch.A. (1975), The Semantic System of Noun Classes in Proto Bantu, Antropological Linguistics 17, 127-138.

Lan, D. (1985), Guns and Rain: Guerillas and Spirit Mediums in Zimbabwe. Berkley: University of California Press.

Palmer, G.B. (2000), Lingüistica cultural. Trad. E. Bernárdez. Madrid: Alianza Editorial.

Wilk-Racięs a, J. (1997), Significación de las palabras: ¿el sistema o la convencionalidad?, Actas del I Congreso Internacional de Semántica, Il, Universidad de la Laguna, 1439-1449.

Wilk-Racięska, J., (cn prensa), El tiempo interno. Una aproximación al aspecto en español. Katowice: Wydawnictwo Uniwersytetu Sląskicgo.

Witherspoon, G. (1977), Language and Art in the Navajo Universe. Ann Arbor: University of Michigan Press. 